\title{
Optofluidic vortex arrays generated by graphene oxide for tweezers, motors and self-assembly
}

\author{
Jiapeng Zheng ${ }^{1}$, Xiaobo Xing ${ }^{1,2}$, Julian Evans ${ }^{3}$ and Sailing $\mathrm{He}^{1,3,4}$
}

\begin{abstract}
Manipulating large numbers of a variety of particles/wires is essential for many lab-on-a-chip technologies. Here we generate a planar array of optofluidic vortices with photothermal gradients from an easy-fabricated graphene oxide (GO) heater to achieve high-throughput and multiform manipulation at low excitation power and low loss. As a tweezer, each vortex can rapidly capture and confine particles without restrictions on shapes and materials. The stiffness of the confinement is easily tuned by adjusting the vortex dimension. As a motor, it can actuate any traps to persistently rotate/spin in clockwise or anti-clockwise mode. As a high-performance 'workshop', this work lays the groundwork for various self-assembly ranging from colloid-based clusters, chains, capsules, shells and ultra-thin films, through particles' surface modification and fusion, to nanowire-based architectures.
\end{abstract}

Furthermore, we can create multiple vortex arrays through fabricating an array of heaters, which enables massively parallel manipulation and distributed operations all on a chip.

NPG Asia Materials (2016) 8, e257; doi:10.1038/am.2016.12; published online 1 April 2016

\section{INTRODUCTION}

In recent decades, particles manipulation in an elaborate lab-on-a-chip system has led to considerable breakthroughs in material synthesis with abbreviated times ${ }^{1,2}$ and detection technology with enhanced sensitivity. ${ }^{3}$ A technique enabling multifunctional manipulation of various nanoparticles is desirable in many lab-on-a-chip applications, but many conventional approaches can only provide limited functions. Optoelectronic tweezers, ${ }^{4}$ electrostatic fields ${ }^{5}$ and sub-wavelength slot waveguides ${ }^{6}$ allow for simple trapping without manipulation. Recently, alternative strategies based on a variety of vortices have been proposed for static trapping and rotation/spinning, which enable massively parallel control for particles. The linear momentum and angular momentum of light vortices have been successfully transferred to particles and caused them to be trapped or spin at the center of the laser beams, which are respectively called as optical tweezers and optical spanners. ${ }^{7-11}$ Surface plasmons vortices generated by some laser-excited plasmonic helical-structures can also provide some potential wells and torques for particles' trapping and rotation. ${ }^{12,13}$ However, these vortex manipulations generally require complex light field gradients or complicated metal structures, and have essentially strong restrictions on their materials and shapes. Furthermore, highenergy excitation sources are necessary to overcome the viscous force coming from the surrounding liquid.
Compared with light vortices and plasmons vortices, microscale fluidic vortices can be a more convenient system for driving particles, resulting from various excitation methods (such as magnetics, electrics and thermal effects), and no restrictions on particles' characteristics (including sizes, materials and shapes). ${ }^{14-23}$ They can be divided into two types according to the operational mechanism. One is through forced convection, such as magnetic field driven rotating nanowires, ${ }^{14}$ laser-driven spinning birefringent spheres $^{15}$ or in expansion chamber. ${ }^{16,17}$ Because of small and limited scale, vortices of this type were generally applied to low throughput manipulation. Abundant experimental cases ${ }^{14,15}$ have shown that individual trapping at the vortex center is stable, but to the best of our knowledge no clear theoretical analysis has been given. The other type is through buoyancy convection, generated by thermal gradient in the liquid from opto-electrical, ${ }^{18-22}$ or plasmonic ${ }^{23}$ heating. Buoyancy convection can flow in the vertical direction with a large and fixed scale, leading to extensive rotation in the vortices and high-throughput aggregation and migration outside the vortices. ${ }^{22}$

In this work, we proposed a new approach for precise manipulation through the third type convection, namely, the thermocapillary convection, which were produced at the liquid level as an array and can be observed more directly (as shown in Figure 1a). Light-driven planar arrays of thermocapillary vortices with controllable dimension

${ }^{1}$ Centre for Optical and Electromagnetic Research, ZJU-SCNU Joint Research Center of Photonics, South China Academy of Advanced Optoelectronics, South China Normal University, Guangzhou, China; ${ }^{2}$ MOE Key Laboratory of Laser Life Science \& Institute of Laser Life Science, College of Biophotonics, South China Normal University, Guangzhou, China; ${ }^{3}$ State Key Laboratory of Modern Optical Instrumentations, Centre for Optical and Electromagnetic Research, JORCEP, Zhejiang University, Hangzhou, China and ${ }^{4}$ Department of Electromagnetic Engineering, JORCEP, School of Electrical Engineering, Royal Institute of Technology, Stockholm, Sweden

Correspondence: Professor S He, Centre for Optical and Electromagnetic Research, ZJU-SCNU Joint Research Center of Photonics, South China Academy of Advanced Optoelectronics, South China Normal University, Guangzhou 510006, China.

E-mail: sailing@jorcep.org

or Professor X Xing, MOE Key Laboratory of Laser Life Science \& Institute of Laser Life Science, College of Biophotonics, South China Normal University, Guangzhou 510631, China.

E-mail: xingxiaobo@scnu.edu.cn

Received 1 October 2015; revised 16 December 2015; accepted 7 January 2016 
a

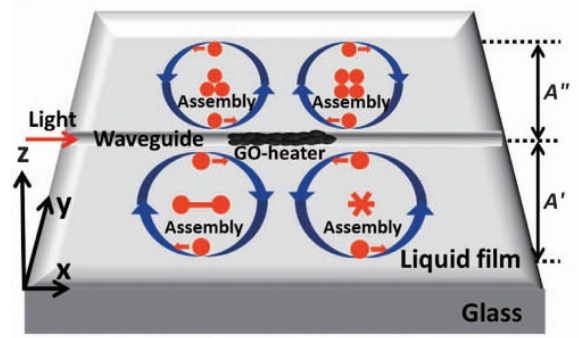

b

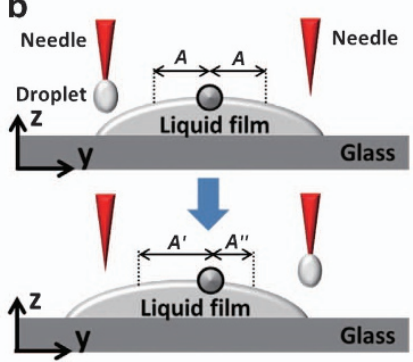

C

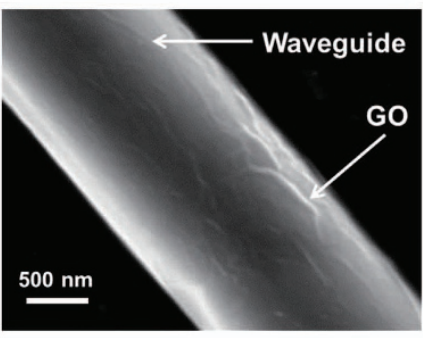

Figure 1 Overview of optofluidic vortices. (a) Configuration of single GO-heater at a liquid-air interface to generate an array of planar vortices. (b) Schematic diagram for increasing or decreasing the dimension of the vortex array by injecting or sucking out droplets. (c) A typical SEM image of a GO-heater made by the droplet approach, illustrating the tight attachment of GO on the waveguide with diameter of $1.2 \mu \mathrm{m}$ because of van der Waals forces.

were applied to achieve both individual and high-throughput manipulation. Thus experimentally, the thermocapillary vortices can stably trap the objects and make them to rotate constantly at the vortex centers with minimal flow velocity. Moreover, various self-assembly of multicomponent-structures including clusters, chains, multilayered capsules, hollow sphere and nanowire-based architectures can be obtained at the vortex centers. The thermocapillary vortices, served as tweezers, motors and 'workshops' for self-assembly, will open up new avenues for multifunctional manipulation at the micro- and nanoscale. Moreover, we have produced multiple vortex arrays from series of graphene oxide (GO) heaters on a waveguide, which enabling a wider range of manipulation by one light excitation and improving energy utilization efficiency. As a type of multispot arrays technologies, ${ }^{24}$ the vortex arrays are expected to achieve simultaneously comparing test and massively parallel manipulation on a chip, as an important analytical tool in material synthesis, medical research and clinical diagnostics. In this paper, we have also theoretically analyzed the velocity fields of the vortices by combining thermodynamics and hydrodynamics. We contributed the possible driving mechanisms to a combination of inertial force (for attracting particle) and viscous force (for rotating particle).

\section{MATERIALS AND METHODS}

\section{Device fabrication}

GO was prepared by oxidizing natural graphite powder based on a modified Hummers method. ${ }^{25}$ As prepared GO was dialyzed to remove the residual salts and acids completely, and the resulting purified GO powder was collected by centrifugation and then dried in the air. GO powder was suspended in ultrapure water $\left(0.5 \mathrm{mg} \mathrm{m}^{-1}\right)$ and exfoliated through ultrasonication in a water bath (KQ218, $60 \mathrm{~W}$ ) for $3 \mathrm{~h}$. As a result, the bulk of GO powder was transformed into GO nanosheets. The GO-heater was fabricated by depositing GO on a waveguide made by drawing a single-mode optical fiber (SMF-28, Corning Inc., New York, NY, USA) using two stepper motors through the flame-heated technique. The experimental details were also shown in Supplementary Methods 1.1 and 1.2.

\section{Experimental procedures}

To produce these vortex arrays, we create thermal gradient using GO heaters, fabricated by combining a silica waveguide (diameter: $0.1-5 \mu \mathrm{m})$ and GO. Supplementary Method 1.1 shows two fabrication methods (deposition approach and droplet approach) with simple and automatic process and without complex microfabrication technique. A typical SEM image of a GO-heater made by droplet approach shown in Figure 1c illustrates the tight attachment of GO on the waveguide due to strong adsorptive capacity of GO. ${ }^{26}$ In the waveguide, light was confined and absorbed in the tube-shaped GO-heater. When the GO-heater works at a liquid-air interface, the temperature variation (because of the heat transfer) of the surrounding liquid induces a corresponding surface tension gradient, and then gives rise to a planar array of vortices owing to the thermocapillary effect. Here $\mathrm{N}, \mathrm{N}$-dimethylformamide (DMF) was used as the surrounding liquid due to the low absorption in near-infrared region. ${ }^{27,28}$ For the convenience of individual and high-throughput manipulations, we control easily the dimension of each vortex, roughly by adjusting the geometry of liquid surface and delicately by tuning the optical power. Figure $1 \mathrm{~b}$ shows the profile view about how we adjust the vortex dimension $(A)$, by injecting or sucking out droplets. Correspondingly, the vortex will have an increased $\left(A^{\prime}\right)$ or decreased dimension $\left(A^{\prime \prime}\right)$. Supplementary Method 1.2 shows the whole process of adjusting vortex dimension (A) from 5 to $200 \mu \mathrm{m}$ by changing the liquid surface and from 60 to $150 \mu \mathrm{m}$ by changing the optical power.

\section{Simulation}

Simulation results were obtained numerically by coupling Navier-Stokes equation, heat-transfer equation and thermocapillary effects, based on COMSOL Multiphysics 4.4 (COMSOL, Stockholm, Sweden).

\section{RESULTS AND DISCUSSION}

\section{Theoretical analysis}

The strong light confinement and high absorption of GO at nearinfrared region ${ }^{29}$ are the key for heat generation. Mode characteristics of the silica waveguide with GO-cladding were analyzed by COMSOL Multiphysics 4.4. At the free-space wavelength of $1550 \mathrm{~nm}$, the silica core with refractive index $n_{1}=1.4457$ and the GO-cladding with large refractive index $\mathrm{n}_{2}=2.6$, and the waveguide was immersed in DMF with refractive index $\mathrm{n}_{3}=1.428$. On the basis of the Helmholtz equation, the optical field distribution of the fundamental mode for such a waveguide with the silica diameter of $2.0 \mu \mathrm{m}$ and the GO-cladding thickness of $1.2 \mu \mathrm{m}$ was shown in Figure 2a. One can see clearly that light was confined in the tube-shaped GO-cladding. Thus, using a fiber laser of low power, the GO-cladding can strongly confine and absorb the optical energy to generate a large enough amount of heat. Moreover, the excellent thermal conductivity ${ }^{26}$ $\left(\sim 600 \mathrm{~W} \mathrm{~m} \mathrm{~m}^{-1} \mathrm{~K}^{-1}\right)$ of GO is helpful to uniform heating. Our analysis shows similar optical field profiles when varying the thickness (Supplementary Figure S5 and Supplementary Method 1.3). Uniform temperature on the GO-heater can be achieved when the thickness is less than $5 \mu \mathrm{m}$ and the length is shorter than $50 \mu \mathrm{m}$.

Excited through a near-infrared light with wavelength band of $1525-1565 \mathrm{~nm}$ and low total power at $40 \mathrm{~mW}$, the GO-heater could concentrate strong evanescent field intensity and release massive heat energy to lead to some local boiling of DMF (boiling temperature: $\sim 425.95 \mathrm{~K}){ }^{27,28}$ When the GO-heater is excited, the temperature distribution in the surrounding DMF is governed by the thermal transfer equation, $\nabla \cdot\left(-k \nabla T+\rho C_{p} T \boldsymbol{u}\right)=Q$, where $k$ is the thermal conductivity $\left(\mathrm{W} \mathrm{m}^{-1} \mathrm{~K}^{-1}\right), T$ is the temperature $(K), \nabla T$ is the 
a
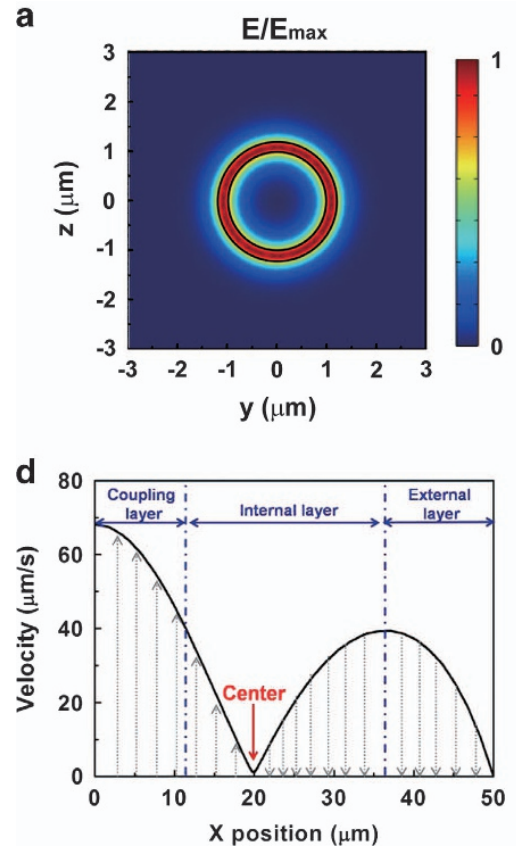

b

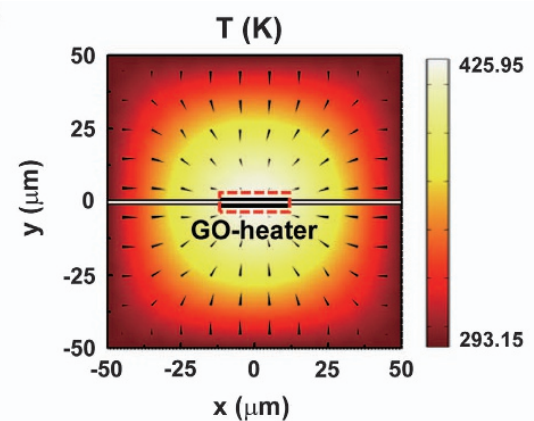

C

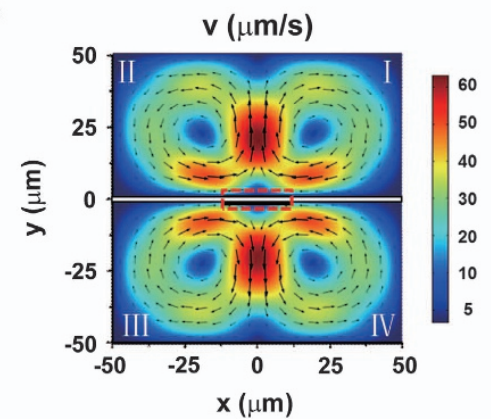

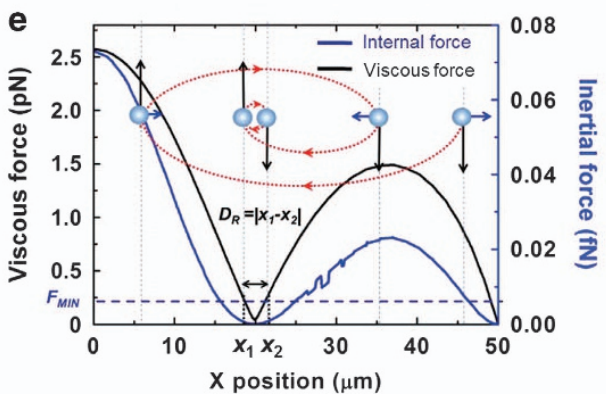

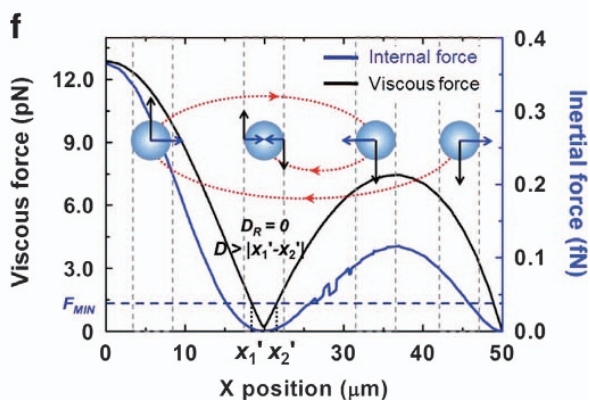

Figure 2 Particle movement in an optofluidic vortex. (a) Optical field distribution of GO-heater on yz-plane. Silica diameter: $2.0 \mu \mathrm{m}$; and GO-cladding thickness: $1.2 \mu \mathrm{m}$. (b) Temperature distribution of a working GO-heater on a liquid surface. The arrows indicate the directions of temperature gradient. (c) Velocity distribution of a working GO-heater, showing four generated equivalent vortices I-IV as an array. There exists a minimal velocity in the vortex I center $(20$ and $22 \mu \mathrm{m})$. (d) The calculated velocity magnitude $(v)$ at various $x$-offsets along the line $y=22 \mu \mathrm{m}$. The velocity magnitude and the direction are indicated by the gray arrows corresponding to vortex I in c. The vortex structure was divided into internal layer $(12 \mu \mathrm{m}<x<36 \mu \mathrm{m})$, external layer $(36 \mu \mathrm{m}<x<50 \mu \mathrm{m})$, and a coupling layer $(0<x<12 \mu \mathrm{m})$ coupled with vortex II. (e and f) Inertial force $F_{l}$ (in the $x$-direction) and viscous force $F_{V}$ (in the $y$ direction) acting over a particle with the diameter of $1 \mu \mathrm{m}$ (e) and $5 \mu \mathrm{m}$ (f). The direction of $F_{I}$ and $F_{V}$ are indicated by the blue and black arrows, respectively. Each particle will be spirally dragged and finally attracted toward the center as indicated by red arrows. Until in the center, its movement is mainly controlled by viscous force and finally rotated along the flow direction. Small one will rotate around the center (e) while large one will spin at the center (f).

temperature gradient, $C_{p}$ is the heat capacity $\left(\mathrm{J} \mathrm{kg}^{-1} \mathrm{~K}^{-1}\right)$ and $Q$ $\left(\mathrm{W} \mathrm{m}^{-3}\right)$ represents a source term, that is, the GO-heater. Because of the existence of temperature distribution, the fluid is capable of yielding convective flow, whose velocity field $\boldsymbol{u}$ and the pressure distribution $p$ can be described by Navier-Stokes equations, $\eta \nabla^{2} \boldsymbol{u}$ $+\rho \boldsymbol{u} \cdot \nabla \boldsymbol{u}+\nabla p=F$ and $\nabla \cdot \boldsymbol{u}=0$, where $\eta$ is the dynamic viscosity $\left(\mathrm{kg} \mathrm{m}^{-1} \mathrm{~s}^{-1}\right), \rho$ is the density $\left(\mathrm{kg} \mathrm{m}^{-3}\right)$ and the $F$ term is a source term representing external forces per unit volume $\left(\mathrm{N} \mathrm{m}^{-3}\right)$. Moreover, for most Newtonian liquids, surface tension decreases linearly with increasing temperature. Therefore, by imposing a temperature gradient on the liquid layer, it will be accompanied by a corresponding surface tension gradient on liquid-gas interface, and a resulting interfacial shear stress which is proportional to the temperature gradient $\nabla T_{\mathrm{S}}$. The difference in interfacial shear stress $\tau_{\mathrm{S}}$ drives flow from regions of low surface tension to regions of high surface tension, opposite to the temperature gradient, as described by $\tau_{\mathrm{S}}=-\sigma_{\mathrm{T}} \cdot \nabla T_{\mathrm{S}}$, where $\sigma_{\mathrm{T}}$ is the temperature coefficient of surface tension.

Simulations using COMSOL Multiphysics 4.4 show the temperature and velocity distribution of vortices I - IV from a GO-heater working on liquid surface with area of $100 \times 100 \mu \mathrm{m}$ (Figures $2 \mathrm{~b}$ and c). Each vortex has a dimension of $\sim 50 \mu \mathrm{m}$ and its flow direction (clockwise or anti-clockwise) is opposite to that of any neighboring one, and in each vortex such as vortex I, there exists a minimal velocity in the vortex center $(20$ and $22 \mu \mathrm{m})$ and velocity gradient perpendicular to the flow velocity and directing towards the center. Further analysis shows similar velocity profiles when varying the boundary area, revealing the stability and flexibility of the vortices system (Supplementary Figure S5 and Supplementary Method 1.3).
Here the driving mechanism is a combination of inertial force (for attracting a particle) and viscous force (for rotating a particle). Vortex centers with minimal flow velocity have been shown to be stable trapping positions for objects by Petit et al., ${ }^{14}$ with abundant experimental cases, but to the best of our knowledge no clear theoretical analysis. In our case, the dynamics may be understood by a combination of viscous force along the flow direction $\left(F_{V}=3 \pi \eta v D\right.$, where $\eta$ denotes fluid viscosity, $v$ denotes the flow velocity and $D$ is the 'effective' diameter of particle), and inertial force directing along the velocity gradient $\left(F_{I}=R e \cdot F_{V}\right.$, where $R e$ denotes Reynolds number).$^{30}$ Taking a line passing through the center, $y=22 \mu \mathrm{m}$ as an example (Figure $2 \mathrm{~d}$ ), $F_{I}$ tends to attract a particle located in the internal or coupling layer toward the center of vortex while repel it away from the vortex when it is in the external layer, and $F_{V}$ is responsible to circulate the particle in pace with the vortex flow. Because of the strong $F_{I}$ in the coupling layer, a particle of $D=1 \mu \mathrm{m}$ even in the external layer could be dragged through a spiral trajectory into the internal layer and finally attracted to the center (red arrow in Figure $2 \mathrm{e})$. Note that the centrifugal force $\left(F_{\mathrm{C}}=m v^{2} / R\right.$, where $m$ denotes particle mass, $v$ denotes the flow velocity and $R$ is rotation radius) is too weak $(<0.001 \mathrm{fN})$ to restrain the attraction. Only if the particle is attracted to the equilibrium position of $\mathrm{x}=x_{1}$ or $x_{2}$ where $F_{I}=F_{\text {Imin }}\left(F_{\text {Imin }}\right.$ denotes the threshold value of inertial force balanced with pressure differences so it fails to attract the particle $\left.{ }^{31}\right)$, the dominating $F_{V}$ will lead to clockwise rotation with diameter $D_{R}$ (that is, $\left.\left|x_{1}-x_{2}\right|\right)$, which can also be expressed as the confinement range (Figure 2e). A bigger particle could experience a diminished rotation diameter and enhanced confinement due to the increased forces. Thus, 
a

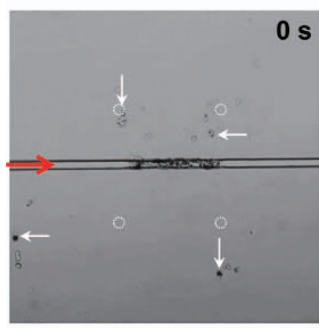

C
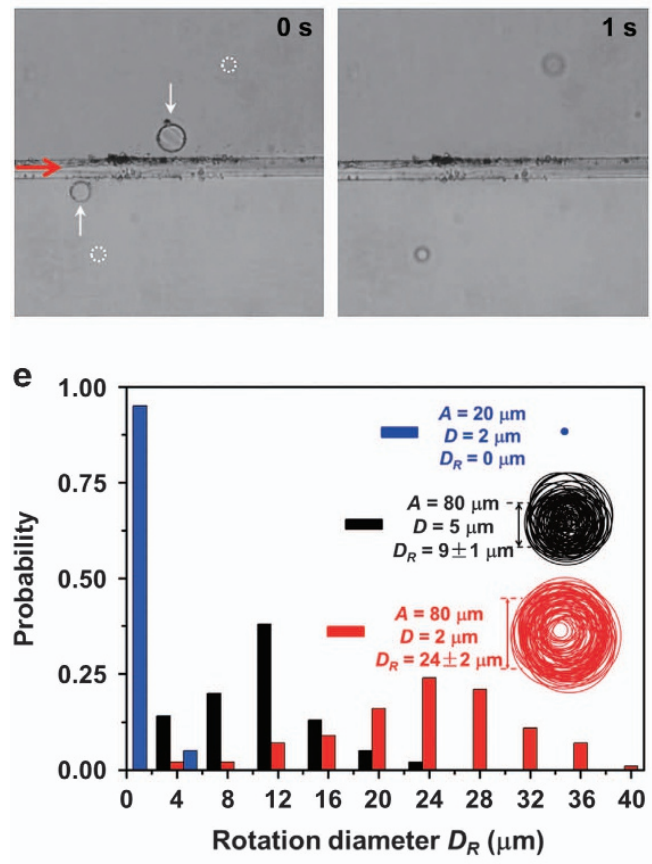

b
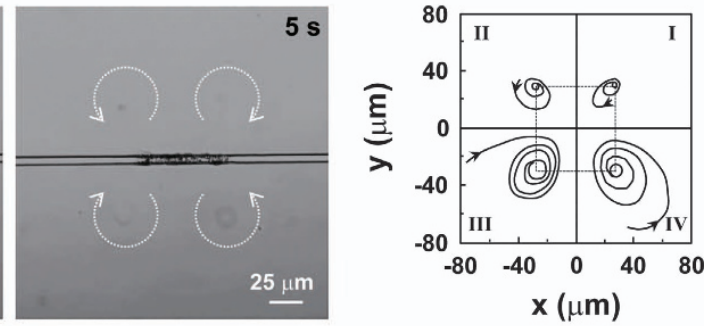

d
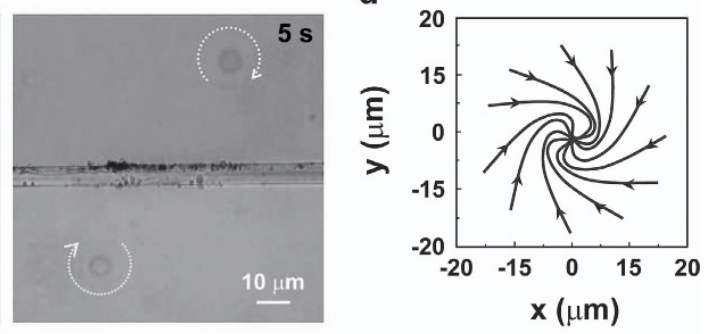

f

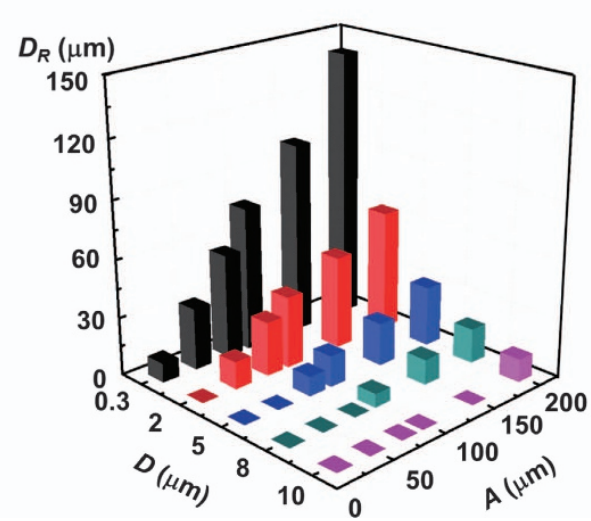

Figure 3 Particle trapping and confinement in optofluidic vortices. (a) Microscope snapshots from Supplementary Video 1 on particles with $D=2$ or $5 \mu \mathrm{m}$ being trapped in vortices I-IV separately with the effective dimension of $A \approx 80 \mu \mathrm{m}$. (b) The corresponding paths for the particles in a undergoing spiral trapping trajectories. (c) Microscope snapshots from Supplementary Video 1 on particles with $D=4$ and $2 \mu \mathrm{m}$ being trapped in vortices I and III, respectively, each with an effective dimension of $A \approx 20 \mu \mathrm{m}$. (d) The particles of $D=2 \mu \mathrm{m}$ in vortex III in c with different original positions were observed to travel through similar paths and finally trapped at the same center. (e) The stiffness of particles confinement can be indirectly indicated from the fluctuations of their circular motion in the vortex. The red path and red histogram correspond to a particle with $D=2 \mu \mathrm{m}$ in vortex IV in a indicating $D_{R}$ of $24 \pm 2 \mu \mathrm{m}$ and $63 \%$ of confinement probability. The black path and black histogram correspond to the case of $D=5 \mu \mathrm{m}$ by vortex I in a $\left(D_{R}=9 \pm 1 \mu \mathrm{m}\right.$, and $82 \%$ of confinement probability). The blue path and blue histogram correspond to the case of $D=2 \mu \mathrm{m}$ by vortex III in c ( $D_{R}=0$, and $98 \%$ of confinement probability). (f) Three-dimensional histogram on the relation between $D, A$ and $D_{R}$. As $A$ decreases or $D$ increases, the rotation diameter is diminished, and finally the rotation will be replaced by the spin with the stiffest confinement. The vortex centers are indicated by white circles.

a particle of $D>\left|x_{1}{ }^{\prime}-x_{2}{ }^{\prime}\right|$ such as $D=5 \mu \mathrm{m}$ would be led to stable and clockwise spin in the vortex center (Figure $2 \mathrm{f}$ ) whose rotation diameter $D_{R}$ can be set as 0 .

\section{Trapping and confining as tweezers}

The vortex array allows for four equilibrium positions in the trapping experiment. We first considered the cases of the particles with $D=2$ or $5 \mu \mathrm{m}$ being captured (see Figure $3 \mathrm{a}$ and Supplementary Video 1 ) and confined in vortices $\mathrm{I}-\mathrm{IV}$ of $A \approx 80 \mu \mathrm{m}$, showing the equivalent mechanical response of each vortex. The corresponding spiral trajectories depicted in Figure $3 \mathrm{~b}$ also show gradually decreasing inertial force for attracting particles and throughout dominating viscous force for transporting particles. Additionally, the particles of $D=2$ and $4 \mu \mathrm{m}$ in the smaller vortices of $A \approx 20 \mu \mathrm{m}$ were observed to experience a shorter spiral trajectory and stay at the center (see Figure $3 \mathrm{c}$ and Supplementary Video 1$)$. The particles $(D=2 \mu \mathrm{m})$ with different original positions were observed to travel through similar paths and finally park at the identical center as depicted in Figure 3d, demonstrating the centrosymmetry of the velocity distribution. Such a tweezer exhibits rapid response time $(<1 s)$ in optical switching. Compared with optical tweezers ${ }^{9}$ and plasmonic tweezers, ${ }^{12}$ which can precisely trap nanoparticles of semiconductors and dielectrics rather than metals, the vortex tweezers have no restrictions on materials or shapes, and thus other materials such as line-shaped Ag-nanowires can also be easily trapped (Supplementary Experiment 2.1). Compared with a waveguide with strong near-field optical forces to transport some matter ${ }^{6}$, the hydrodynamic force in the vortex can withstand near-field optical forces from a microfiber tip at a similar power level, suggesting that the confinement is stable and tight enough to resist disturbances from external electromagnetic field (Supplementary Experiment 2.2). Furthermore, the vortices were observed to move with the GO-heater, and meanwhile the particles were continuously trapped and translated by the mobile vortices, exhibiting a similar flexibility as optical tweezers (Supplementary Experiment 2.3). 


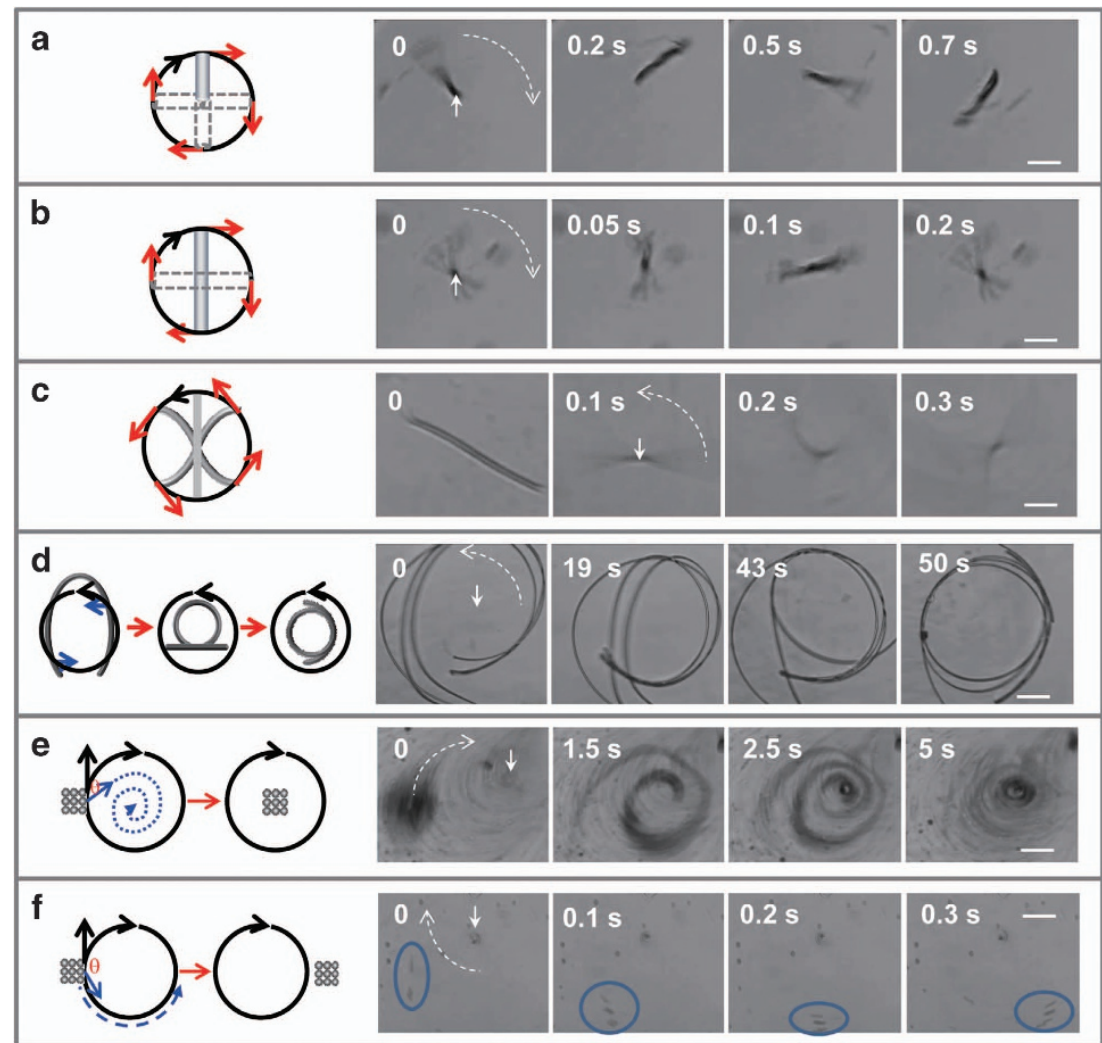

Figure 4 Hydrodynamic micro-motor for rotation/spin/manipulation of nanowires/particles in optofluidic vortices. (a-d) Schematic pictures (left) and microscope snapshots from Supplementary Videos 2 (right), showing that the motor provides a permanent torque, which can compel a silica rod $(D \approx 2 \mu \mathrm{m}$ and length: $23 \mu \mathrm{m}$ ) to spin about one of its ends at $\sim 1 \mathrm{~Hz}$ frequency (in a vortex of $A \approx 20 \mu \mathrm{m}$, a) or on its center at $\sim 5 \mathrm{~Hz}$ frequency (in a vortex of $A \approx 80 \mu \mathrm{m}, \mathbf{b}$ ), or cause the elastic deformation of a spinning polymer nanofiber ( $D \approx 780 \mathrm{~nm}$, length: $45 \mu \mathrm{m}$ ) in a vortex of $A \approx 150 \mu \mathrm{m}$ (c), or even fold-bent nanowires $(D \approx 780 \mathrm{~nm})$ into micro-loops with diameter of $45 \mu \mathrm{m}$ (vortex: $A \approx 150 \mu \mathrm{m}, \mathbf{d})$. (e, f) The motor can trap requisite particles such as a handful of nanoparticles $(D \approx 300 \mathrm{~nm}$, vortex: $A \approx 50 \mu \mathrm{m}, \mathrm{e})$, or repel unnecessary samples such as a handful of nanoparticles $(D \approx 2 \mu \mathrm{m}$, vortex: $A \approx 50 \mu \mathrm{m}$, f), depending on their direction $\theta<90^{\circ}$ or $\theta>90^{\circ}$. The vortex centers and rotation directions are indicated by solid arrows and dotted arrows. Scale bars, $10 \mu \mathrm{m}$.

Because of the confined potential well and certain flows around the center, a naturally stable vortex trap is always accompanied by a persistent rotation/spin. The red paths in Figure $3 \mathrm{e}$ illustrate the circulatory movement of a trapped particle $(D=2 \mu \mathrm{m}$, in vortex IV of Figure $3 \mathrm{a}$ ) with frequency $\sim 5 \mathrm{~Hz}$, showing the rotation diameter with strong fluctuations. The probability distribution of the rotation diameter, obtained from a period of $60 \mathrm{~s}$ at $5 \mathrm{~Hz}$ and depicted by the red histogram in Figure $3 \mathrm{e}$, also shows the maximum probability located at $24 \pm 2 \mu \mathrm{m}$ (the diameter of confinement region $D_{R}$ ), and the particle has a probability of $63 \%$ to stay inside the confinement region. The black and blue data in Figure $3 \mathrm{e}$ also illustrate the confinement states of Figure $3 \mathrm{a}\left(D=5 \mu \mathrm{m}, A \approx 80 \mu \mathrm{m}, D_{R}=9 \pm 1 \mu \mathrm{m}\right.$ and $82 \%$ of confinement probability) and Figure $3 \mathrm{c}(D=2 \mu \mathrm{m}, A \approx 20 \mu \mathrm{m}$, $D_{R}=0$ and $98 \%$ of confinement probability), respectively, showing that spinning particles will be subjected to the stiffest and stablest confinement in the vortex. Dependences of $D_{R}$ on $D$ and $A$ were also considered for a complete quantitative description of $D_{R}$ shown in Figure 3f, illustrating that a particle's rotation is shrinking accompanied by reducing $A$ or increasing $D$, and finally substituted for spin.

\section{Rotating or spinning as motors}

In addition to confinement and tweezers, the vortex array can also serve as quad-core driving motors generating persistent torques. Light-driven motors have previously been achieved from angular momentum of illuminating light $t^{8,15}$ and symmetrical plasmonic structures. ${ }^{11,32}$ In our case, the optical motors do not require designed structure or predetermined light and have flexible dimension (Supplementary Experiment 2.4). The mode (clockwise or anticlockwise) of light-driven motors has previously been controlled by using different circular polarizations ${ }^{15}$ or varying the wavelength ${ }^{32}$ of the illumination. In our motor, two modes can be actualized simultaneously in a vortex array, where clockwise mode exists in vortices I and III and anti-clockwise mode exists in vortices II and IV. As an active agent for rotational actuators, our motor is capable of compelling a silica rod to spin about one end (Figure 4a), or on its center (Figure 4b). Supplementary Figure S9 also shows how to switch these spin modes by changing the dimension of vortices. A polymer rod spinning on its center may even cause deformation (Figure $4 \mathrm{c}$ ). Driven by a rotation mode, we first proposed nanowires immersed in the vortex to be automatically bent and then folded together and finally twirled into nanowire-based resonators (Figure 4d), which is expected to be useful for microscale-lasers. ${ }^{33}$

Besides rotational actuators, our motor can exert influence on particles outside the vortex and attempting to enter at an angle $\theta$ with the rotation direction. The vortex tends to permit them immediately into the internal layer when $\theta<90^{\circ}$, but forbid entry and compel them to swim around the external layer when $\theta>90^{\circ}$. Figures $4 \mathrm{e}$ and $\mathrm{f}$ illustrate how the particles can be one by one swum into and away from the vortex, respectively. The former is conducive to appending 


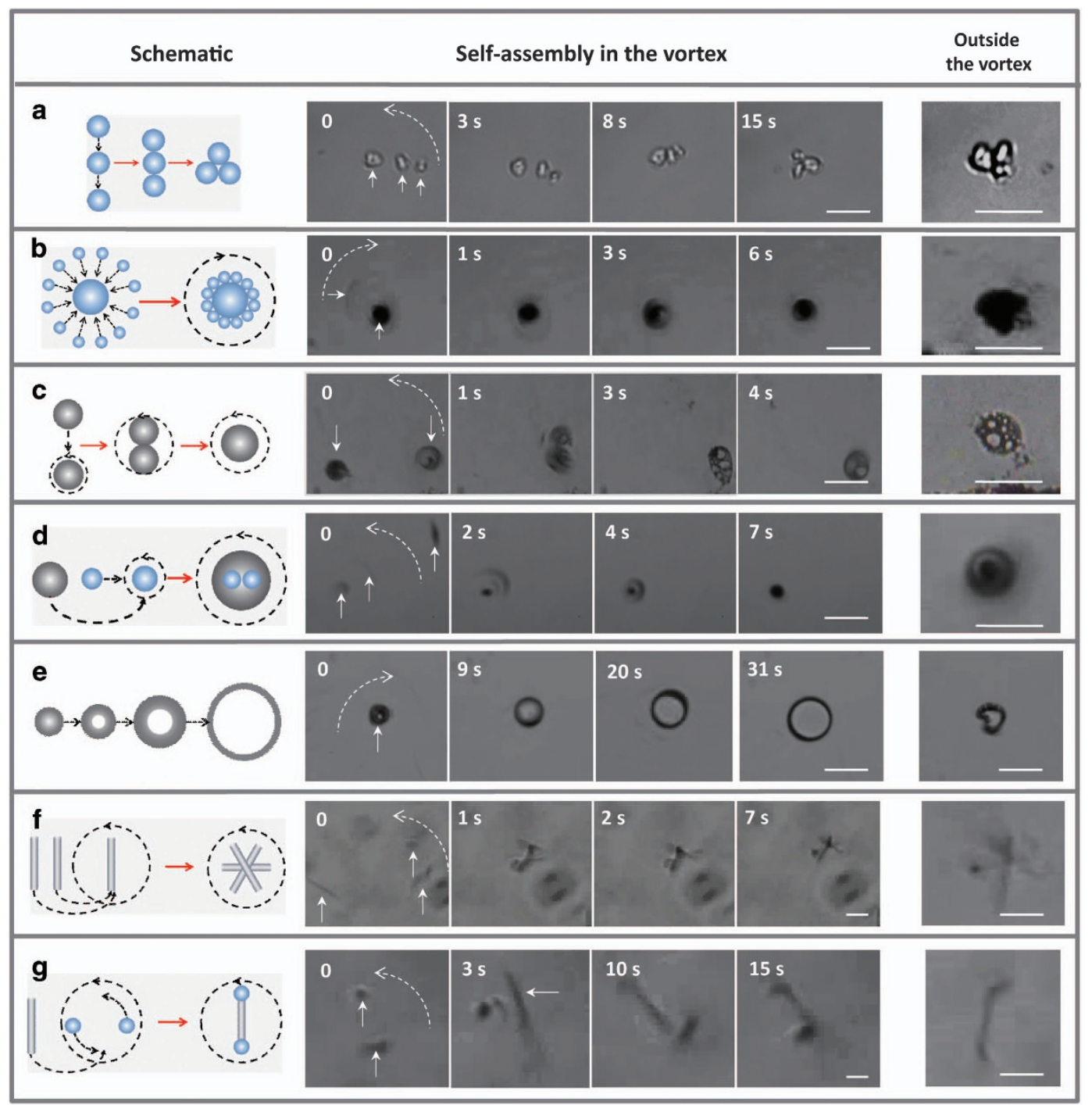

Figure 5 Various types of self-assembly for particles/nanowires in optofluidic vortices. Schematic pictures (left) and microscope snapshots from Supplementary Videos 3 (middle) and enlarged final structures outside the vortices (right panels), showing seven types of self-assembly for multicomponent particles/nanowires. (a) Three particles of different sizes $(D \approx 2,3$ and $4 \mu \mathrm{m})$ accumulated in the vortex center $(A \approx 80 \mu \mathrm{m})$, forming block-type structures such as a linear-chain and a triangular cluster. (b) Nanoparticles $(D \approx 300 \mathrm{~nm})$ were collectively introduced to decorate a target $(D \approx 2 \mu \mathrm{m})$ for surface modification (vortex: $A \approx 20 \mu \mathrm{m})$. (c) Two droplet $(D \approx 2 \mu \mathrm{m})$ collided together at the center (vortex: $A \approx 20 \mu \mathrm{m}$ ) and fused into a new particle. (d) $A$ droplet $(D \approx 2 \mu \mathrm{m})$ was introduced to isolate the trapped targets $(D \approx 1 \mu \mathrm{m})$ from the background liquid, forming a capsule (vortex: $A \approx 20 \mu \mathrm{m})$. (e) $\mathrm{A}$ solid polymer sphere $(D \approx 2 \mu \mathrm{m})$ was translated into a hollow particle, and then inflated to form a polymer shell (vortex: $A \approx 20 \mu \mathrm{m})$. (f) Three Ag nanowires $(D \approx 300 \mathrm{~nm}$ and length: $10 \mu \mathrm{m}$ ) were captured sequentially and finally constituted a structure resembling symbol '*' (vortex: $A \approx 50 \mu \mathrm{m})$. (g) Two particles $(D \approx 2 \mu \mathrm{m})$ and a nanowire $(D \approx 300 \mathrm{~nm}$ and length: $20 \mu \mathrm{m})$ finally led to a stable dumbbell-shaped structure (vortex: $A \approx 80 \mu \mathrm{m})$. The assembling components and rotation directions are indicated by solid arrows and dotted arrows. Scale bars, $5 \mu \mathrm{m}$.

requisite particles, while the latter repels unnecessary samples. The details of Figure 4 can also be viewed in Supplementary Video 2.

\section{'Workshops' for self-assembly}

By integrating the functions of tweezers and motors, individual vortex is also capable of trapping nanoparticles of various materials, then leading to their local interactions by rotation/spin, and orderly selfassembling into an organized structure or pattern. The resulted assemblies can still maintain a stable state by the local van der Waals action among the components even after the vortex is weakened by the diminished power or disappeared by turning off the light. Intended multicomponent-structures based on polystyrene particles were easily designed by controlling the vortex dimension, the order of the particles to be trapped and the total number of the particles to be assembled. Figure 5a shows the formation of linear- and block-type structures from accumulating particles, for a case of three particles to be rotated on different orbits with uniform angular velocity and then collide one by one in the end into a linear-chain, which was finally transformed into a triangular cluster. Particles of different sizes and materials can be introduced and the assembly number and order can be controlled, so that other building blocks of different shapes such as quadrangle, pentagon and plum blossom ${ }^{34,35}$ are achievable, which is potentially applied in colloidal particles synthesis. Figure 5b shows coating of small particles on a target particle. After trapping a large target particle, a handful of nanoparticles were collectively introduced to surround it, and the spinning motion supported uniform coating. 
a
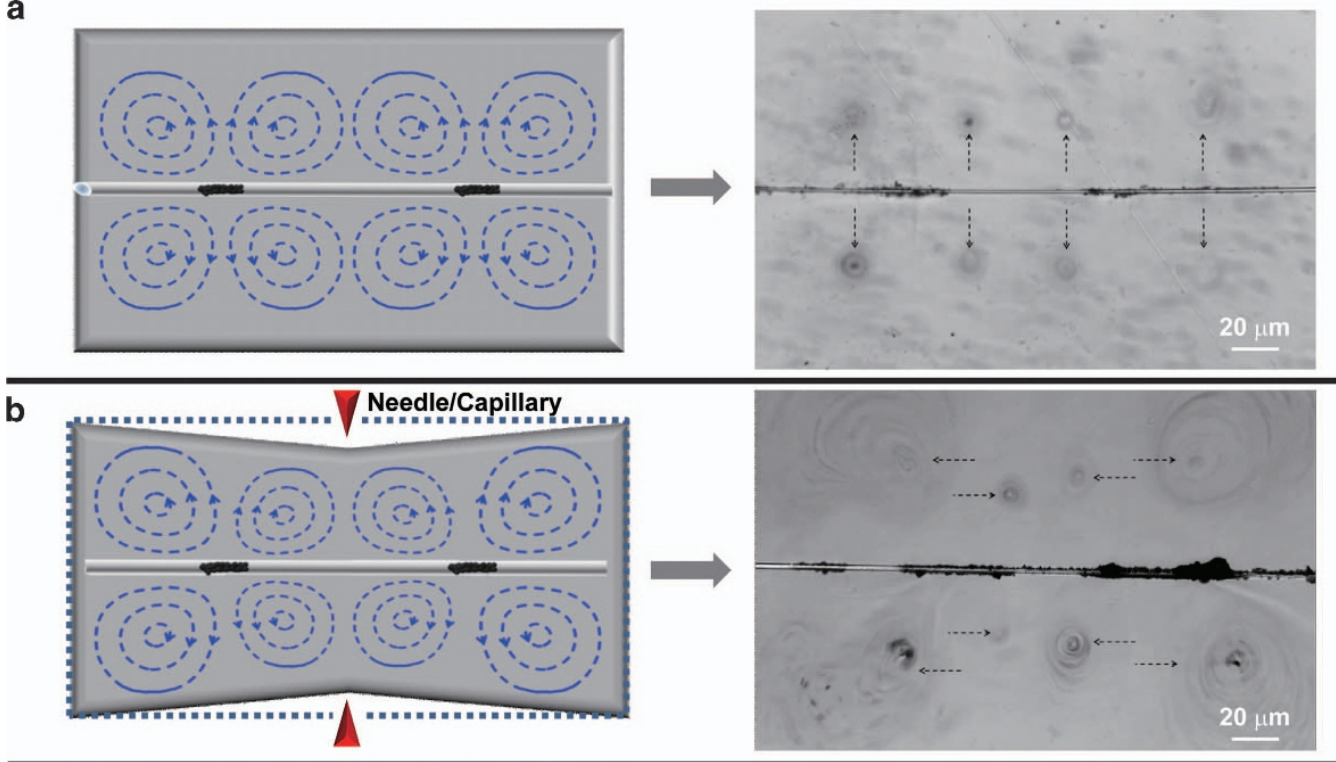

c
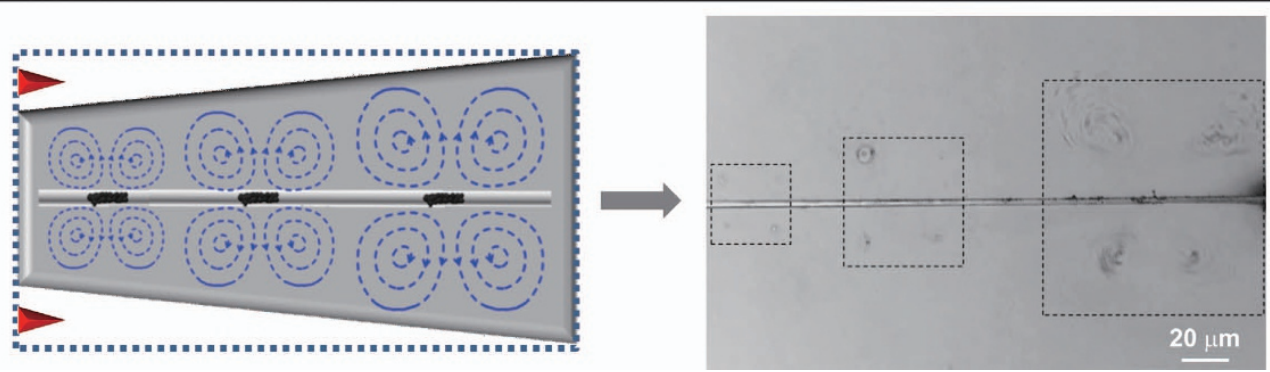

Figure 6 Scalability of the cascaded arrays of optofluidic vortices. (a) Generation of eight vortices ( $A \approx 50 \mu \mathrm{m}$, indicated by black arrows) around two sections of heaters to confine various particles $(D=2-10 \mu \mathrm{m})$ to rotate/spin, which can offer eight trapping centers or driving motors. (b) Using two needles or capillaries to suck out droplets between two heaters, we can generate eight vortices (indicated by black arrows) with the first and forth columns of larger size $(A \approx 100 \mu \mathrm{m})$ for accommodating all the unwanted particles and the middle vortices of smaller size $(A \approx 50 \mu \mathrm{m})$ for capturing the desired particles $(D=2-10 \mu \mathrm{m})$. (c) Using two needles or capillaries to suck out droplets on the first heaters, we can generate twelve analogous vortices around three heaters (indicated by the black frames), which were separately arranged to spin particles with $D \approx 2 \mu \mathrm{m}$, spin particles with $D \approx 10 \mu \mathrm{m}$ and assemble particles with $D \approx 1 \mu \mathrm{m}$. The blue dotted lines indicate the original geometry of liquid surface.

Various surface modifications can be assembled automatically by decorating different nanomaterials and capsules layer by layer for biomedical applications. ${ }^{36}$

We also demonstrated the assembly based on soft materials such as phospholipid membranes, immiscible droplets and two-phase systems. Polystyrene droplets could collide together on the vortex center, fuse into an elongated patch and subsequently fold into one sphere (Figure 5c), showing a potentially strategy for multiple-components coalescence. Figure 5d shows particles packaged into a capsule for drug-delivery research. ${ }^{37} \mathrm{~A}$ polystyrene droplet was introduced to collectively isolate the trapped targets from the background liquid and the spin was also conducive to a complete package. The number of the layers and components are uniquely determined by the arrangement of the traps. In addition, we show that a vortex can transform a spinning solid polymer sphere into a hollow sphere owing to the centrifugal effect. Resembling an elastic bubble, the spinning hollow sphere can also be inflated under the injection of solvent to form a polymer shell (Figure 5e). The injected material also depends on the surrounding solvent, and the sphere diameter and shell thickness can be accurately controlled with the spinning time. By means of the elasticity, the hollow sphere can serve as a novel vehicle useful in, for example, ultra-sensitive resistive pressure sensor. ${ }^{38}$ Without any chemical pre-treatment, ${ }^{35}$ all the assemblies proceeded over a period of $<1 \mathrm{~min}$, which was significantly shorter than that of analogous technologies. ${ }^{36}$ It is expected to obtain more stable assembly coming from chemical reaction of the components in the vortices for a short time.

In addition to particles, the vortex also enables the construction of nanowire architectures. Figure $5 \mathrm{f}$ shows nanowires assembled into complex heterostructures that may function as active photonic devices. ${ }^{39,40}$ Three Ag nanowires were captured sequentially in a specified order and spun on their centers, and finally constituted a structure resembling symbol ' $*$ ' due to different orientations. As shown in Figure 5g, after trapping two particles and a nanowire, their continual rotation and collisions finally led to a stable dumbbell-shaped structure, which represents a promising device for nanophotonics. ${ }^{41}$ The details of Figure 5 can also be viewed in Supplementary Video 3. Furthermore, the vortices can control nanoparticles for the high-throughput fabrication of ultra-thin film materials of controllable size (Supplementary Experiment 2.5).

\section{Cascaded arrays of optofluidic vortices}

By fabricating a row of heaters (each of which is capable of producing a vortex array), we show multiple 'workshops' on the same waveguide, which is expected to achieve a sequence of optofluidic manipulation 
through reasonable allocation. The dimension of the vortex arrays can be properly adjusted as shown in Figure 6. To demonstrate their operational capacity, we designed eight vortices of uniform size around two heaters and let it work on various stiffly confining and continually rotating/spinning particles (Figure 6a). We also devised eight vortices, including the first and forth columns of larger size capable of accommodating all the unwanted particles and the middle vortices of smaller size in charge of capturing the desired objects, which can defend desired objects from other pollution in a mixture (Figure 6b). We also produced twelve vortices on three heaters, arranged into three groups according to the size of 20,60 and $100 \mu \mathrm{m}$, and let them spin particles with $D \approx 2 \mu \mathrm{m}$, spin particles with $D \approx 10 \mu \mathrm{m}$ and assemble particles with $D \approx 1 \mu \mathrm{m}$, respectively (Figure 6c). The details of Figure 6 can also be viewed in Supplementary Video 4. Because of the decreasing optical power along waveguide, a silica waveguide holds a maximum of three GO heaters based on photothermal effect. By cascading multiple waveguides, more arrays of optofluidic vortices are expected to enable massively parallel manipulation on a chip.

\section{CONCLUSION}

We have demonstrated cascaded arrays of scalable optofluidic vortices for multifunctional manipulation, enabling active trapping and stiff confinement as tweezers, providing constant torque as motors without any symmetrical structure or spiral light, and allowing various self-assemblies for nanowires, particles and droplets. At the operation wavelength, other liquids such as nutrient solution with low absorption can be used to yield vortices for bio-sample (such as bacteria and cells) manipulations. This technique could be further combined with some other techniques such as external electric/magnetic fields to discriminate and sort different samples. Through appropriate assignment, arrays of vortices lend themselves to a series of 'workshops' for expanded and integrated multi-functions. This will enable large-scale and simultaneously multiple experimental operations in an elaborate lab-on-a-chip system such as microfluidic control, materials science and engineering, chemistry synthesis and analysis, biopharmaceutics, microelectronic engineering, photonics and nanoscience.

\section{CONFLICT OF INTEREST}

The authors declare no conflict of interest.

\section{ACKNOWLEDGEMENTS}

We thank Prof. Da Xing from Education Ministry's Key Laboratory of Laser Life Science \& Institute of Laser Life Science, College of Biophotonics, South China Normal University, for his support in experiments. This work is partially supported by Guangdong Innovative Research Team Program (Grant 201001D0104799318), the National Natural Science Foundation of China (Nos. 91233208, 61177077, 61178062 and 81371877), Guangdong Natural Science Foundation (2013B090500123 and 2014A030313432), the National High Technology Research and Development Program (863 Program) of China (No. 2012AA012201), Guangdong Excellent Doctoral Dissertation Funded Project (SYBZZXM201126), Swedish VR grant (\# 621-2011-4620) and AOARD, and the Scientific Research Foundation of Graduate School of South China Normal University (No. 2015lkxm11).

1 Valencia, P. M., Farokhzad, O. C., Karnik, R. \& Langer, R. Microfluidic technologies for accelerating the clinical translation of nanoparticles. Nat. Nanotechnol. 7, 623-629 (2012).

2 Elvira, K. S., Casadevall i Solvas, X., Wootton, R. C. R. \& deMello, A. J. The past, present and potential for microfluidic reactor technology in chemical synthesis. Nat. Chem. 5, 905-915 (2013).
3 deMello, A. J. Control and detection of chemical reactions in microfluidic systems. Nature 442, 394-402 (2006).

4 Chiou, P. Y., Ohta, A. T. \& Wu, M. C. Massively parallel manipulation of single cells and microparticles using optical images. Nature 436, 370-372 (2005).

5 Krishnan, M., Mojarad, N., Kukura, P. \& Sandoghdar, V. Geometry-induced electrostatic trapping of nanometric objects in a fluid. Nature 467, 692-695 (2010).

6 Yang, A. H., Moore, S. D., Schmidt, B. S., Klug, M., Lipson, M. \& Erickson, D. Optical manipulation of nanoparticles and biomolecules in sub-wavelength slot waveguides. Nature 457, 71-75 (2009).

7 Grier, D. G. A revolution in optical manipulation. Nature 424, 810-816 (2003).

8 Paterson, L., MacDonald, M. P., Arlt, J., Sibbett, W., Bryant, P. E. \& Dholakia, K. Controlled rotation of optically trapped microscopic particles. Science 292, 912-914 (2001).

9 Ashkin, A. Acceleration and trapping of particles by radiation pressure. Phys. Rev. Lett. 24, 156-159 (1970).

$10 \mathrm{He}$, H., Friese, M. E. J., Heckenberg, N. R. \& Rubinsztein-Dunlop, H. Direct observation of transfer of angular momentum to absorptive particles from a laser beam with a phase singularity. Phys. Rev. Lett. 75, 826-829 (1995).

11 Padgett, M. \& Bowman, R. Tweezers with a twist. Nat. Photon. 5, 343-348 (2011).

12 Juan, M. L., Righini, M. \& Quidant, R. Plasmon nano-optical tweezers. Nat. Photon. 5, 349-356 (2011).

13 Tsai, W. Y., Huang, J. S. \& Huang, C. B. Selective trapping or rotation of isotropic dielectric microparticles by optical near field in a plasmonic archimedes spiral. Nano Lett. 14, 547-552 (2014).

14 Petit, T., Zhang, L., Peyer, K. E., Kratochvil, B. E. \& Nelson, B. J. Selective trapping and manipulation of microscale objects using mobile microvortices. Nano Lett. 12, 156-160 (2012).

15 Wu, T., Nieminen, T. A., Mohanty, S., Miotke, J., Meyer, R. L., Rubinsztein-Dunlop, H. \& Berns, M. W. A photon-driven micromotor can direct nerve fibre growth. Nat. Photon. 6, 62-67 (2012).

16 Mach, A. J., Kim, J. H., Arshi, A., Hur, S. C. \& Di Carlo, D. Automated cellular sample preparation using a centrifuge-on-a-chip. Lab Chip 11, 2827-2834 (2011).

17 Wang, X., Zhou, J. \& Papautsky, I. Vortex-aided inertial microfluidic device for continuous particle separation with high size-selectivity, efficiency, and purity. Biomicrofluidics 7, 307-315 (2013).

18 Kumar, A., Williams, S. J. \& Wereley, S. T. Experiments on opto-electrically generated microfluidic vortices. Microfluid. Nanofluid. 6, 637-646 (2009).

19 Mizuno, A., Nishioka, M., Ohno, Y. \& Dascalescu, L. D. Liquid microvortex generated around a laser focal point in an intense high-frequency electric field. IEEE Trans. Ind. Appl. 31, 464-468 (1995).

20 Williams, S. J., Kumar, A., Green, N. G. \& Wereley, S. T. A simple, optically induced electrokinetic method to concentrate and pattern nanoparticles. Nanoscale $\mathbf{1}$, 133-137 (2009).

21 Park, C. \& Wereley, S. T. Rapid generation and manipulation of microfluidic vortex flows induced by AC electrokinetics with optical illumination. Lab Chip 13, 1289-1294 (2013).

22 Williams, S. J., Kumar, A. \& Wereley, S. T. Electrokinetic patterning of colloidal particles with optical landscapes. Lab Chip 8, 1879-1882 (2008).

23 Roxworthy, B. J., Bhuiya, A. M., Vanka, S. P. \& Toussaint, K. C. Jr Understanding and controlling plasmon-induced convection. Nat. Commun. 5, 155-164 (2014).

24 Howbrook, D. N., van der Valk, A. M., O'Shaughnessy, M. C., Sarker, D. K., Baker, S. C. \& Lloyd, A. W. Developments in microarray technologies. Drug Discov. Today 8, 642-651 (2003).

25 Kovtyukhova, N. I., Ollivier, P. J., Martin, B. R., Mallouk, T. E., Chizhik, S. A., Buzaneva, E. V. \& Gorchinskiy, A. D. Layer-by-layer assembly of ultrathin composite films from micron-sized graphite oxide sheets and polycations. Chem. Mater. 11, 771-778 (1999).

26 Zhu, Y., Murali, S., Cai, W., Li, X., Suk, J. W., Potts, J. R. \& Ruoff, R. S. Graphene and graphene oxide: synthesis, properties, and applications. Adv. Mater. 22, 3906-3924 (2010).

27 Xing, X., Zheng, J., Sun, C., Li, F., Zhu, D., Lei, L., Cai, X. \& Wu, T. Graphene oxide-deposited microfiber: a new photothermal device for various microbubble generation. Opt. Express 21, 31862-31871 (2013).

28 Xing, X., Zheng, J., Li, F., Sun, C., Cai, X., Zhu, D., Lei, L., Wu, T., Zhou, B., Evans, J. \& Chen, Z. Dynamic behaviors of approximately ellipsoidal microbubbles photothermally generated by a graphene oxide-microheater. Sci. Rep. 4, 6086 (2014).

29 Li, M., Yang, X., Ren, J., Qu, K. \& Qu, X. Phototherapy: using graphene oxide high nearinfrared absorbance for photothermal treatment of alzheimer's disease. Adv. Mater. 24, 1621-1621 (2012).

30 Di Carlo, D. Inertial microfluidics. Lab Chip 9, 3038-3046 (2009).

$31 \mathrm{Xu}$, R., Xin, H. \& Li, B. Massive assembly and migration of nanoparticles by laser-induced vortex flows. Appl. Phys. Lett. 103, 014102 (2013).

32 Liu, M., Zentgraf, T., Liu, Y., Bartal, G. \& Zhang, X. Light-driven nanoscale plasmonic motors. Nat. Nanotechnol. 5, 570-573 (2010).

33 Xiao, Y., Meng, C., Wang, P., Ye, Y., Yu, H., Wang, S., Gu, F., Dai, L. \& Tong, L. Singlenanowire single-mode laser. Nano Lett. 11, 1122-1126 (2011).

34 Demirörs, A. F., Pillai, P. P., Kowalczyk, B. \& Grzybowski, B. A. Colloidal assembly directed by virtual magnetic moulds. Nature 503, 99-103 (2013). 
35 Wang, Y., Wang, Y., Breed, D. R., Manoharan, V. N., Feng, L., Hollingsworth, A. D., Weck, M. \& Pine, D. J. Colloids with valence and specific directional bonding. Nature 491, 51-55 (2012)

36 Cortez, C., Tomaskovic-Crook, E., Johnston, A. P., Radt, B., Cody, S. H., Scott, A. M., Nice, E. C., Heath, J. K. \& Caruso, F. Targeting and uptake of multilayered particles to colorectal cancer cells. Adv. Mater. 18, 1998-2003 (2006).

37 Loretta, L., Rivera-Gil, P., Abbasi, A. Z., Ochs, M., Ganas, C., Zins, I., Sönnichsen, C. \& Parak, W. J. LbL multilayer capsules: recent progress and future outlook for their use in life sciences. Nanoscale 2, 458-467 (2010).

38 Pan, L., Chortos, A., Yu, G., Wang, Y., Isaacson, S., Allen, R., Shi, Y., Dauskardt, R. \& Bao, Z. An ultra-sensitive resistive pressure sensor based on hollow-sphere microstructure induced elasticity in conducting polymer film. Nat. Commun. 5, 3002 (2014).

39 Pauzauskie, P. J., Radenovic, A., Trepagnier, E., Shroff, H., Yang, P. \& Liphardt, J. Optical trapping and integration of semiconductor nanowire assemblies in water. Nat. Mater. 5, 97-101 (2006).

40 Jamshidi, A., Pauzauskie, P. J., Schuck, P. J., Ohta, A. T., Chiou, P. Y., Chou, J., Yang, P. \& Wu, M. C. Dynamic manipulation and separation of individual semiconducting and metallic nanowires. Nat. Photon. 2, 85-89 (2008).
41 Heiss, M., Fontana, Y., Gustafsson, A., Wüst, G., Magen, C., O’Regan, D. D. Luo, J. W., Ketterer, B., Conesa-Boj, S., Kuhlmann, A. V., Houel, J., Russo-Averchi, E., Morante, J. R., Cantoni, M., Marzari, N., Arbiol, J. Zunger, A., Warburton, R. J. \& Fontcuberta i Morral, A. Self-assembled quantum dots in a nanowire system for quantum photonics. Nat. Mater. 12 439-444 (2013).

(c) (i) This work is licensed under a Creative Commons Attribution 4.0 International License. The images or other third party material in this article are included in the article's Creative Commons license, unless indicated otherwise in the credit line; if the material is not included under the Creative Commons license, users will need to obtain permission from the license holder to reproduce the material. To view a copy of this license, visit http://creativecommons.org/licenses/by/4.0/

Supplementary Information accompanies the paper on the NPG Asia Materials website (http://www.nature.com/am) 